\title{
Fuentes bibliográficas de Arte y Uso de Arquitectura de Fray Lorenzo de San Nicolás
}

\author{
Pedro antonio López Gayarre
}

Cuando en el año $1639^{1}$ sale de la estampa la primera parte del que ha sido calificado por $G$. Kubler como «el mejor tratado sobre instrucción arquitectónica escrito jamás" ${ }^{2}$, su autor, el Agustino Recoleto Fray Lorenzo de San Nicolás, cumplía con un proyecto encaminado a dotar al mundo de la construcción de un instrumento de trabajo preeminentemente práctico y que recogía todos los problemas que podian surgir a lo largo de un proceso de edificación.

El cáracter de manual de esta primera parte del Arte y Uso de Architectura condicionará en gran manera el recurso que el autor hará de las principales fuentes, limitando su uso a lo largo de la obra en aras de la claridad e inteligencia de lo expuesto. Así el conocimiento de obras, que se pone de manifiesto a lo largo de la Segunda Parte (1655), con la exposición del sistema de órdenes arquitectónicos de los principales tratadistas, no aparece en esta primera.

Roza lo increible los equivocos que las primeras ediciones de los Tomos I y || del Arte y Uso de Architectura han provocado y de los que no se ha salvado el que escribe estas líneas: si con buen criterio Juan J. Martín González en el prólogo a la edición facsimil (Valencia, 1989. Ed. Albatros) coloca como fecha de edición del primer volumen 1639, correspondiente a la tasa y fe de erratas a pesar de datarse las licencias en 1633, en el segundo volumen se propone como fecha 1664, cuando la fe de erratas y tasa última es de 1665 .

Quede pues claro desde ahora y a pesar de las fechas citadas anteriormente que las fechas de edición de las primeras impresiones son 1639 y 1665.

2 Kubler, G., “Arquitectura de los siglos XVII y XVII" Tom. XIV. Ars Hispaniae. Madrid, 1957. Pág. 80 . 
Ya la portada, a imitación de la edición española de la Regola... de Vignola $^{3}$, o de Los Cuatro Libros de Arquitectura de Palladio ${ }^{4}$, nos acerca a la concepción que Fray Lorenzo tiene de la práctica arquitectónica y que se refrenda a lo largo del capitulo primero, en el que se considera a la Aritmética y a la Geometria como los pilares básicos de la formación del Arquitecto ${ }^{5}$.

Curiosamente esta manera de entender la Arquitectura choca frontalmente con la idea vitruviana, máxima autoridad para Fray Lorenzo, del arquitecto como resumen del saber universal y de la Arquitectura como ciencia humanistica por excelencia, compendio de todas las demás y sin cuyo conocimiento es imposible su desarrollo ${ }^{6}$.

Fray Lorenzo, alejándose de cualquier especulación gratuita, dedicará los primeros capítulos a mostrar las principales reglas aritméticas; suma, resta, multiplicación, división, quebrados, reglas de tres y de compañia y raices cuadradas y los principales y elementales conceptos geométricos ?

Con este corto bagaje instrumental Fray Lorenzo será capaz, a lo largo del tratado, de presentar y resolver todos y cada uno de los problemas de la edificación del siglo, considerándolo suficiente y sobrado para desarrollar su labor ${ }^{8}$.

${ }^{3}$ Vignola lacome (Barozzi) de, "Regla de las cinco órdenes de Architectura de... Agora de nueuo traduzido de Toscano en Romance por Patritio Caxesi. Florentino, pintor y criado de su Mag. Dirigido al Principe Nuestro Señor. En Madrid, en casa del Autor, en la calle de la Chruz. 1593.

4 Palladio, andrea, I quatro libri dell'Architectura. Venecia, 1570. Por Dominico de Franceschi.

5 San Nicolas, Fray Lorenzo de, Arte y Uso de Architectura, Madrid 1639. Tomo I. Capitulo I: "Trata del Architectura Arismetica y Geometría; de su necesidad; y de como conuienen entre si y de sus primeros inuentores". Fol. 1.

Utilizo para las citas la Edición facsimil de Ed. Albatros: 1639-1665. Realizada en Vanlencia en 1989.

${ }^{6}$ Vitruvio Polion, M., Los diez libros de Architectura de..., traducidos del Latín y comentados por Don Joseph Ortiz y Sanz. Presbitero. Madrid, 1787. Imprenta Real. Libro I. capitulo 1: (Refiriendose a las cualidades y saberes del arquitecto) "Será instruido en las Buenas Letras, diestro en el Dibuxo, hábil en la Geometria, inteligente en la Optica, instruido en la Aritmética, versado en la Historia, Filósofo, Médico, Juriconsulto, y Astrólogo".

San NiCOLAS, Fray Lorenzo de, obra citada. Capitulos 2 al 20, páginas: fols. $2 v$ al 26. Tomo 1.

${ }^{8}$ Ibídem, Tomo I, Cap. XV, fol. 21: "De todas las reglas hasta aqui dichas tiene necesidad el Architecto de saberlas bien como adelante conocerá. No trato de mas rayces que ay, por bastar lo dicho, que como al principio en el Prólogo dixe, solo de Arismética y Geometria tomare lo necesario para el Architecto: mas el que quisiere saber mas abundantemente la Arismética lea desde el primero hasta el dezimo libro de Moya, y cumplirá su deseo, que este autor escriuio desta Arte mucho y bien y assi puede emplearse en su 
En cuanto a Aritmética se refiere, la obra utilizada para la redacción de los primeros capítulos y nombrada como "la Aritmética de Moya" no puede ser otra sino la Arithmética Práctica y Speculativa de Juan Pérez de Moya ${ }^{9}$; obra que fue editada por primera vez en el año 1562 y de cuyo éxito lo dicen todo las dieciseis ediciones que se conocen y que se reparten a lo largo del siglo XVII y XVIII hasta la última de $1798^{10}$.

Juan Pérez de Moya es ante todo y en la Arithmética... se pone de manifiesto, un vulgarizador de la Ciencia y principalmente de las Matemáticas, que logra llegar al "gran público" a través de una exposición clara y didáctica de los principales saberes matemáticos.

La Geometría tomada y propuesta como modelo es la de Euclides, expuesta en la primera edición de la Primera Parte en cinco breves capitulos en los que de forma clara y concisa se resumen las "definiciones, peticiones, conceptos o comunes sentencias, cosas pertenecientes a líneas y valor de los ángulos".

La importancia del geómetra griego en la época se comprueba por las sucesivas ediciones del Arte y Uso; pues si ya en la primera edición de la Segunda Parte aparecen las traducciones de los Libros Quinto y Séptimo (1665) ${ }^{11}$, en la segunda impresión de la Primera Parte, aparecen en lugar de los cuatro primeros capitulos-resumen de las definiciones, éstas de manera completa, además de la traducción íntegra del Primer Libro de Euclides que se añade al final ${ }^{12}$.

leyenda, pues della sacará noticia de mucho oculto a su ingenio. Mas lo hasta aqui escrito, bien entendido y obrado, como despues obraremos, bastará para lo que en el Arte se pueda ofrecer".

9 Perez de Moya, Juan, Arithmética Práctica y Speculativa, Salamanca, 1562. Matias Gast.

10 Bonet Correa, A., Garcia Melero, J. E., Dieguez Patao, S., lorenzo fornies, S., Bibliografia de Arquitectura, Ingenieria y Urbanismo en España. 1498-1880.

Recogen estos autores ediciones de los siguientes años: Salamanca, 1562; Granada, 1590; Madrid, 1598; Madrid, 1609; Alcalá, 1619; Madrid, 1624; Madrid, 1625; Madrid, 1643; Madrid, 1652; Madrid, 1663; Madrid, 1672; Madrid, 1675; Zaragoza, 1694, Madrid, 1615; Barcelona, 1705; Madrid, 1723; Madrid, 1729; Madrid, 1745; Madrid, 1752; Madrid, 1761; Madrid, 1776; Madrid, 1784; Madrid, 1798.

iSan Nicolas, F. Lorenzo de, Segunda parte del Arte y Uso Architectura. Dedicada al desamparo que padeció mi redentor Jesuchristo las tres horas que estubo viuo enclabado en el Arbol de la Cruz. Con el Quinto y Séptimo libros de Euclides traducidos de latin en Romance. Y las medida difíciles de Bouedas y de las superficies y pies cúbicos de Pichinas. Con las ordenanzas de La Imperial Ciudad de Toledo aprobadas y confirmadas por la Cesarea Magd. del Sr. Emperador Carlos V de gloriosa memoria. Compuesto por... Agustino descalzo Architecto y Maestro de obras natural de la muy noble y coronada Villa de Madrid. Madrid, 1665.

12 SAN NiCOLAS, F. Lorenzo de, "Segunda ynpresión de la primera parte del arte y uso 
En este especial tratamiento de la Geometría euclidica no hacia Fray Lorenzo sino seguir la tendencia de la época y buena prueba de ello es el interés que en el siglo anterior despertó la figura de Euclides en los centros cercanos a la Corte y en la Academia de Matemáticas impulsada por Juan de Herrera y que hizo notar desde sus primeros pasos la necesidad de contar con la traducción de los libros del megarense ${ }^{13}$.

Las traducciones, a partir de las versiones latinas del Padre Cristobal Clavio S. J., alemán, son del cosmógrago portugués Antonio de Náxera (Libros I Y V) y Juan de la Rocha, matemático y ligado a la corte como el anterior (Traducción del VII).

Los tratados de Geometria contemporáneos apenas tienen presencia; sólo son citados el dominico español fallecido en 1567, Fray Juan de Ortega del que debe ser el Tratado sutilisimo de aritmética y geometria ${ }^{14}$ y Andrés de Céspedes, en realidad Garcia de Céspedes y su Tratado de Instrumentos de Geometria ${ }^{15}$, publicado en 1606.

de architectura, dirixido al patriarca San Joseph, con el primer libro de Euclides traducido de latin en Romance compuesto por el padre... Agustino descalzo y maestro de obras y arquitecto, natural de la muy noble i coronada villa de Madrid. Madrid 1667.

${ }^{13}$ La primera traducción al castellano de la Geometria de Euclides es: "Los seis libros primeros de la Geometria. Traducidos en lengua española por Rodrigo Camorano, Astrólogo y Mathemático, y Cathedrático de Cosmographia por su Magestad en la casa de la Contratación de Sevilla..." Sevilla, 1576. En Bonet y otros, obra citada.

La segunda traducción está ligada a la academia de Matemáticas de Herrera: “La Perspectiva y especularia de Euclides. Traduzidas en vulgar castellano, y dirigidas a la S.C.R.M. del Rey don Phelippe nuestro señor. Por Pedro Ambrosio Onderiz su criado ..." Madrid, 1585.

En: Bonet y otros, obra citada.

Sobre el tema de la Academia de Herrera, véase

Checa Cremades. F.: "Los ingenieros del renacimiento y la mentalidad clasicista, en el Catálogo de la Exposición Herrera y el Clasicismo. Valladolid, 1986.

${ }^{14}$ San NicolaS, F. Lorenzo de, obra citada, Vol. I, Cap LXXVII.: La primera edición de la obra de Fray Juan Ortega lleva por titulo: "Siguese una compusición de la Arte de la Arismética y Juntamente de geometria. Lyón, 1512.

Se recogen en BONET y otros, obra citada dos ediciones latinas: Roma 1515 y Messina 1525. además de ediciones castellanas de: Sevilla 1534, 1537, 1542 y 1552 y Granada 1563, bajo el título, con algunas variantes, de: Tratado sutilissimo de Arithmética y Geometria.

La edición granadina, realiza cuatro años antes de la muerte del Dominico Fray Juan de Ortega recoge como colotón una parte de la obra de Juan Perez de Moya: "Libro de cuenta que tracta de la quatro Reglas generales de Arthmetica practica, por numeros enteros. y quebrados, y de reducciones de monedas destos reynos de Castilla, con un razonamiento sobre la misma facultad..." Toledo, 1559.

${ }^{15}$ San Nicolas, Fray Lorenzo de, obra citada, Vol. I. Cap. LXVII. Garcia de Cespedes, Andres: Libro de Instrumentos nuevos de Geometria y muy necessarios para medir distancias y alturas sin que intervengan numeros como se demuestra en la práctica. Madrid, 1606. 
Al tratar en el capitulo LXIII de los principales problemas de fortificación se citan y recomiendan al lector los tratados de Diego González de Medina y Barba: Examen de fortificación, Madrid, 1599 y del Capitán Cristobal de Rojas, Teórica y Práctica de fortificación conforme a medidas $y$ defensas destos tiempos, repartidas en tres partes, Madrid, $1598^{16}$.

Es curioso hacer notar, en cuanto a este último tratado, la coincidencia con Fray Lorenzo sobre la necesidad del conocimiento de la Geometria y la Aritmética básica, como apoyos fundamentales y saberes instrumentales con los que es posible resolver todos los problemas de fortificación. Hay un claro paralelismo entre los dos tratados al abordar los saberes que debe dominar el arquitecto y el ingeniero y que serán la base de su curriculum académico ${ }^{17}$.

Como apuntábamos anteriormente el tratado de Vitruvio conforma y llena de contenido todo el Arte y Uso de Arquitectura y así Fray Lorenzo afirma:

"Ninguno se maraville de como de ordinario cito más a Vitruvio que a otros autores, habiendo tanto escrito de esta materia, pues no es la causa el no haberlos visto, sino que todo cuanto hay escrito de Arquitectura, es de este Autor; y asi Sebastiano lo que halló fuera de los preceptos de Vitruvio lo reprueba. A este Autor se le debe mucho, por haber dado mucha luz del Arte, y asi confesaré lo que fuere suyo en la ocasión que se ofreciere, excusando el nombrar a otros, pues ellos se valieron de la autoridad de este Autor para autorizar la suya, como yo me valdré en lo que fuere suyo" ${ }^{18}$.

La misma idea de la supremacia de Vitruvio sobre los demás tratadistas se encuentra de forma reiterada a lo largo de la obra:

is Sobre la vida y obras del Capitan Cristobal de Rojas, véase:

- Mariategui, Eduardo de, El Capitan Cristobal de Rojas Ingeniero Militar del siglo xvi. Madrid, 1985. Reedición del estudio publicado por primera vez en 1880 . Editado por el CEHOPU. Acompana a este estudio: Tres tratados sobre fortificación. del Capitan Cristobal de Rojas.

Sobre tratados de fortificación, veasé:

- Camara Munoz, Alicia: "Tratados de arquitectura militar en España. Siglos XVI y XVIr». Rev. Goya núm. 156, mayo-junio 1980, pag. 338-345.

- "La arquitectura militar y los ingenieros de la monarquia española: aspectos de una profesión (1530-1650)". Rev. de la Universidad Complutense, num. 3. 1981, pags. 225-269.

17 Rojas, Cristobal de, Teorica y practica de fortificacion.. Madrid, 1598. Cap. 1: "De las cosas que son necesarias para la fortificacion».

${ }^{1}$ San Nicolas, Fray Lorenzo de, obra citada, Tom 1, Cap. I. Fol. 2v. 
"... y porque es cosa graue, me valdré de la autoridad de Vitruvio; a quien los más de los Architectos siguen..." 19.

«... He puesto esta disposición de disminuyr la coluna, por ser la que mas comunmente siguen todos; mas como me precio de tan obseruador de los preceptos de Vitruvio, deseando hallar regla con la qual se pueda disminuir, no solo el deseo pasado, sino también con las particulares medidas deste Autor, que sea facil le halle..." ${ }^{20}$.

«... mas el Arte nunca ata las manos al Architecto, aunque a los preceptos deste Autor todos deuieramos estar sujetos" ${ }^{21}$.

En el capítulo VI del Segundo volúmen del Arte y uso... se nos da a conocer las versiones utilizadas que son:

«I dieci libri dell'architettura di $M$. Vitrubio tradutti et comentati da Monsignor Barbaro eletto patriarca d'Aquileggia", Venecia, 1556. Francesco Marcolini ${ }^{22}$.

De Architectura dividido en diez libros, traduzidos de Latín en Castellano por Miguel de Urrea, Alcalá de Henares, 1582. Juan Gracián ${ }^{23}$.

Expresamente el autor manifiesta el no haber tenido acceso a las versiones "originales" latinas:

"No sé que sea la causa que estos dos que traducen a Vitrubio de una lengua a otra, hablan en diferentes capítulos y en diferentes libros de esta orden, como por acá no hemos visto los originales del Vitrubio, hemonos de valer de 10 traducido" ${ }^{24}$.

En realidad el primer contacto con las ideas vitruvianas se habia realizado a través del libro de Diego de Sagredo: Medidas del Romano necessarias a los oficiales que quieren seguir las formaciones de las

19 Ibidem. Tom. I, Cap. XXV. Fol. 32.

${ }^{20}$ Ibidem. Tom. I, Cap. XXXII. Fol. $40 \mathrm{v}$.

${ }^{21}$ Ibidem. Tom. I, Cap. XXXVI. Fol. 56.

22. Ibidem. Tom. II. Cap. VI. Fol., pág. 22. Recogido en:

Wiebenson, Dora, Los tratados de Arquitectura. De Alberti a Ledoux. Madrid, 1988, pag. 68. Se realizaron reediciones de esta obra en Venecia en los años: 1567, 1567 latina, 1584. 1629 y 1641 .

${ }_{23}$ Ibidem. Tom. II, Cap. VI, Pág. 22. Recogido en:

BONET y otros, obra citada, pág. 144.

${ }_{24}$ Ibidem. Tom. II. Cap. VI, pág. 22. 
Basas, colunas, Capiteles y otras pieças de los edificios antiguos. Toledo, 1526, Ramón de Petrás; como en el cap. 27 del Vol. II afirma el Recoleto:

"De treze años empeçe a estudiar en él, y empeço en mi la afición desta facultad: su título es medidas del Romano Vitrubio".

que parece indicar la utilización de la edición de $1549^{25}$. Aunque el libro de Alberti ${ }^{26}$ es citado reiteradamente, son los tratados de Serlio y Vignola los que sirven como complemento ideal a los problemas no resueltos por Vitruvio:

Asi en el capítulo XXXIII de la primera parte ante la inexistencia de las medidas del orden toscano, contenidas en el desaparecido último libro de Vitruvio, Fray Lorenzo escribe:

"..., mas yo haré aqué deseño (sic) aprouechandome de la autoridad de Sebastiano, en quanto a las proporciones, y el ornato de la de Biñola, que en uno y otro los dos diferencian".

Sobre la autoridad de los tres autores, Vitruvio, Serlio y Vignola descansa toda la teoria arquitectónica vertida a lo largo de esta primera parte: también conoce y utiliza dos ediciones diferentes de la obra del boloñés: la latina de Juan Carlos Carraceno datada en $1569^{27}$ y la cas-

25 SAgredo, Diego de, "Medidas del romano o Uitruvio nuevamente impresas y añadidas muchas pieças necesarias a los oficiales..." Toledo, 1549. Juan de Ayala. Reedición en 1564.

Recogidos en: BONET y otros, obra citada, pág. 122-125

25 Alberti, Leone Battista, De re aedificatoria. Florencia, 1485. Nicolo di Lorenzo Alemanno.

-: Los Diez Libros de Architectura, de León Baptista Alberto. Traducidos de Latín en Romance. Madrid, 1582. Alonso Gómez.

Recogidos en: WIEBEnSON, D., obra citada, pág. 47. y en BONET y otros, obra citada, pág. 56-57.

27 SERLIO, Sebastiano. : "Regole generali di architetura sopra le cinque maniere degli edifici, cio è thoscano, dórico, iónico, corinthio et composito, con gli essempi dell'antiquita. che, per la magior parte concordano con la dottrina di Vitruvio", Venecia, 1537. Francesco Marcolini. Edición latina con traducción de Juan Carlos Carraceno de 1569. En: WIEBENSON, D., obra citada, pág. 164 . 
tellana de Francisco de Villalpando ${ }^{28}$; mientras que al no decir nada, lo lógico es que utilice alguna de las diferentes impresiones realizadas con la versión castellana de Patricio Caxesi del tratado de Jacomo Barozzi ${ }^{29}$.

Serlio y Vignola son los complementos ideales de los pasajes equívocos de Vitrubio o de aquellas partes, caso de la adecuación del modo arquitectónico, que necesita de una reelaboración adaptada a las necesidades del templo cristiano ${ }^{30}$.

Destaca Fray Lorenzo la claridad como la cualidad más importante de la «Regola delli cinque ordini...»:

"En lo poco que escriue, y demuestra este Autor declara con breuedad lo que otros Autores no hazen en mucho escrito, y assi confiesso merece toda alabanza".

"A mi ver este autor dió mucho lustre a las cinco órdenes porque sus adornos son muy ajustados, y propiamente conuienen para los Ensambladores, Plateros, y Pintores, porque usa de miembros más delgados que otros Autores..." ${ }^{31}$.

Esta triada de grandes tratadistas llena, completándose entre si, toda la primera parte de la obra, siendo las referencias a otros tratados conocidos escasas y puntuales.

Hay que destacar la cita que en el capitulo XXII de esta primera parte se hace del libro del Padre Martín Esteban, dedicado a uno de los problemas más queridos por la tratadistica de la época: la reconstrucción arqueológica ideal del desaparecido templo de Salomón como modelo

${ }^{28}$ Serlo. Sebastiano: "Tercer y cuarto libro de Architectura de... Boloñés. En los quales se trata de las maneras de como se pueden adornar los hedificios con los exemplos de las antigüedades. Agora nuevamente traduzido de Toscano en Romance Castellano por Francisco Villalpando Architecto. Dirigido al muy alto y muy poderoso..." Toledo, 1552. Ivan de Ayala. REd. 1563, 1573.

29 Ademas de la edición de 1593, "en casa del autor", existe otra edición del mismo año realizada "en casa Vicencio Carducho" y sucesivas en Madrid, 1597, 1619. 1651. 1658, 1698, 1702, 1722, 1760, 1764 y 1768, además de diferentes compendios y apendices a la obra original.

30 Veáse sobre el problema del modo arquitectónico: de

MARIAS. Fernando: Orden y Modo en la Arquitectura Española, en la versión española 1983.

Forssman, Erik. : Dórico, Jonico, Corintio en la Arquitectura del Renamiento, Madrid.

${ }^{31}$ San Nicol.AS, Fray Lorenzo de, obra citada, Tom. II, Cap. 39, pag. 127 y Cap. 40, pág. 129. 
directamente inspirado por la divinidad y por lo tanto arquetipo del templo cristiano. El Compendio del Rico Aparato y hermosa Architectura del Templo de Salomon, y de la magestad y grandeza del Rey, sacado de la Sagrada Escriptura conforme a la esposición de graves interpretes. Alcalá de Henares, 1615. Juan Gracián; es, como su título indica, un compendio de los escritos anteriores de los jesuitas Prado y Villalpando junto a otros autores que especulan sobre el tema ${ }^{32}$.

Bien distinta es la segunda parte del Arte y Uso de Architectura. correspondiendo a una concepción muy alejada de la primera y uno de cuyos primeros objetivos se expresa en el primer capítulo:

«... para que los discipulos a poca costa, y trabajo de sus maestros lo vengan a ser. $Y$ como para serlo tengan necesidad de rebolver, $y$ mirar los libros que ay escritos desta facultad, y no todos los Maestros los tienen, o por no poder mas, o por no alcançarlos. Aquí pretendo hazer de todos un cuerpo, dando sus medidas de cada uno en quanto a sus cinco ordenes con sus distribuciones y medidas, para que en este tratado vea lo que cada uno dize, y valiendose de la forma y modo de las molduras demostradas en en el capítulo treinta y uno del Arte y uso de Architectura y de los que aqui demostraré: y como aquí fuere leyendo de alli, y de aquí irlo sacando, y obrando acabada la parte de la orden, sea Basa, o Chapitel, o Alquitraue, o Friso, o Cornisa, avra traçado la orden que quisiere de Arquitectura, según el Autor que leyere, he de dar demostración de las cinco ordenes de cada uno, que yo no pretendo copiar los libros a los Autores, sino dezir lo que dize cada uno para que el rnancebo por este medio vea to que todos dizen..." ${ }^{33}$.

Da la impresión que junto a las razones confesadas por Fray Lorenzo al presentar la sucesión de tratados, existe la oculta necesidad de

32 Prado. Jeronimo del y Villalpando, Juan Bautista.: «Hieronymi Pradi et loannis Baptistae Villalpandi e Societate lesu in Ezechielem Explanationes et Apparatus Urbis, ac Tem. pli Hierosolymitani, Commentariis et Imaginibus illustratus. Opus tribus tomis distinctum..." Romae, 1596-1605.

Sobre el tema de la reconstrucción arqueológica del templo de Salomón. veáse:

- Ramifez, J. A., Construcciones ilusorias. Arquitecturas descritas arquitecturas pintadas. Madrid, 1983. págs 113-213.

- "Guarino Guarini. Fray Juan Ricci y el Orden Salomónico entero" Goya. núm. 160. Ene-feb, 1981, pags. 202-211.

- Rodriguez G. de Ceballos, "Juan de Herrera y los jesuitas Villalpando, Valeriani, Ruiz, Tolosa", AHSI. Vol. XXXV, Roma. 1966.

- TAYLOR, R., El padre Villalpando (1522-1608) y sus ideas esteticas. Academia, 1952.

- "Hermetism and mystical architecture in the Society of Jesus" en Barcque Art. The Jesuit contribution. Fordham University Press. Nueva York, 1972, pags. 63-97.

33 San Nicolas, F. LoRenzo de, obra citada, t. II. Cap. I. págs 1-2. 
demostrar unos conocimientos puestos en tela de juicio por las objeciones que Pedro de la Peña realiza a la primera parte del Arte y Uso de Arquitectura y a las que se da cumplida respuesta en los capítulos segundo a quinto de la segunda parte por orden expresa del "Consejo Real".

Asi la segunda parte se convierte en una profusa reseña de las medidas y molduras utilizadas en los diversos órdenes por los principales tratadistas y que nos permiten conocer el amplio conocimiento que de ellos tiene el Agustino Recoleto. Sin embargo, la postura de Fray Lorenzo es de realizar una mera exposición de cada uno de ellos, antes que de asimilar su doctrina, como ocurría con los libros citados en la primera parte, utilizados todos ellos como argumento de autoridad de sus concepciones arquitectónicas.

El análisis pormenorizado se centra en primer lugar en los tratados ya citados de Vitruvio, Serlio, Vignola, Palladio y Sagredo a los que se vienen a añadir los siguientes ${ }^{34}$ :

- Viola Zanini, Giusepe: Della architectura di Giusepe Viola Zanini padovano pittore et architetto libri due, Padua, 1626, Franceso Bolzetta ${ }^{35}$.

- Cataneo, Pietro: La architettura di Pietro Cataneo Senese, Venecia, 1567. Figlioli di Aldo ${ }^{36}$.

- Labacco, Antonio: Libro d'Antonio Labacco appartenente a L'architetture nel qual si figurano alcune notabili antiquita di Roma, Roma, 1552. Casa Nostra. Numerosas ediciones posteriores $^{37}$.

- Rusconi, G. Antonio: Della architettura, di Gio, Antonio Rusconi con centosessanta figura dissegnate dal Medesimo. Secondo i precetti de Vitruvio, e chiarezza e brevitá dichiarate. Libri Dieci. Venecia, 1590. Giolito ${ }^{38}$.

${ }^{34}$ Ibidem. Dedica a Vitruvio los caps. 6 al 9, a Serlio del 10 al 14 , a Palladio del 15 al 20, a Sagredo del 27 al 31 bajo el título de Picardo y Campeso, a Vignola del 39 al 44.

${ }_{35}$ Ibidem. Caps 21 al 25, págs 82 a 94 . En WIEBENSON, D., obra citada, pág. 88.

${ }^{36}$ Ibidem. Cap 26, pág. 95. En WieBenson, D., obra citada, pág. 71

37 Ibidem. Cap. 27, pags 95 a 96. En WIEBENSON, D., obra citada, pág. 66.

38 Ibidem. Cap. 33, págs. 114 a 116. En WIEBENSON, D.. obra citada, pág. 68. 
- Arfe y Villafañe, loan de: De varia commensuración para la esculptura y Architectura, Sevilia, 1585. Andrea Pescioni y Juan de León. Existen ediciones de: Madrid, 1675, 1736, 1763, 1773, 1795 y $1806^{39}$.

- Scamozzi, Vicenzo: Dell'ldea dell architettura universale di Vincenzo Scamozi divisa in X. Libri, Venecia, $1615^{40}$.

Con excepción de los libros de Cataneo, Labacco y Rusconi, el esquema es siempre el mismo: relación de los libros, capítulos y temas que abarca cada tratado para a continuación realizar una minuciosa descripción de los elementos que utiliza cada autor para componer los cinco órdenes clásicos. Con Cataneo, Labacco y Rusconi se limita a describir someramente su contenido.

El esfuerzo descriptivo de Fray Lorenzo, debido a la ausencia de grabados que ilustren tan prolija relación, produce en el lector una impresión muy alejada de los fines didácticos perseguidos, suponiendo para "un mancebo" no iniciado en el arte unas dificultades no demasiado complicado de imaginar ${ }^{41}$.

Sin embargo la ausencia de grabados referidos a la composición de los ordenes no es total, ya que ante las graves dificultades de edición de una obra suficientemente ilustrada y comprendiendo la necesidad de las imágenes, Fray Lorenzo opta por presentar un dibujo por cada uno de los autores con un sólo de los órdenes. Esta selección de modelos, dignos de ser trasladados a las planchas de imprenta encarnan en buena medida las preferencias de nuestro autor de forma elocuente; asi el toscano de Vitruvio, el dórico de Serlio, el jónico de Palladio, el corintio de Vignola y el compuesto de Scamozzi representan los módelos realzados por vía de la imagen gráfica, en la segunda parte del Arte y Uso de

${ }^{39}$ Ibidem. Cap. 34 a 38, págs. 116 a 127. En BONET y otr., obra citada, págs. 60-62.

40 Ibidem. Cap. 45 a 49, págs. 155 a 179. En WIEBENSON, D., obra citada, pág. 83.

${ }^{41}$ Ibidem. Cap. I, pág 2.: Viene de la nota (33): “... y no ay que marauillar el que trate esto sin estampa, sino solo de cinco Autores de cada uno de una orden, estampando de los mejores, que no seré el primero que aya impreso sin estampa ninguna; pues Leon Bautista, Alberto escrivio diez libros de Arquitectura, que andan en un cuerpo, y en ninguno ay estampa de las ordenes, sino solo Theorica...".

Asi dedicará un capitulo a analizar el contenido del libro de Alberti (26), thajo el título: "Trata de algunos libros que tratan de Arquitectura sin demonstraciones de las cinco órdenes". Cap. 32, págs. 112-113. 
Arquitectura, el núcleo de tratados que componen el "cuerpo principal» de doctrina arquitectónica de Fray Lorenzo de San Nicolás ${ }^{42}$.

Al finalizar esta labor no resiste el tratadista la tentación de mostrar los superiores conocimientos que tiene de otras muchas obras sobre la materia:

"De doze autores he sacado lo que ellos dizen cada uno de las cinco órdenes, y pudiera valerme para instruir al práctico Arquitecto político de los preceptos, reglas, y máximas de que se valieron lorge Agricola, Alconsio, Galaso, Alguiso, Iuan Andro Vecio de Cerçeau, Tullio Vellino, Daniel Bárbaro, Cosme Bartolo, Cesar, Cesarino, lacbo Lantero, Eduardo Lupecino, Francisco Montemelino, Crispin de Paz y Guillermo Philander, comentado a Vitruvio, Teodosio Tripolita, Gofredo Torino, Iuan Bautista Villalpando. Benedicto Arias Montano. Tulto Vulteyo, Iuan Bautista Zancho, Dominico Fontana, en su libro del Obilisco Baticano, el Marqués de Cusano Don Garcia de Barrionueuo en su Panegírico, dedicado a Don Pedro Fernandez de Castro, Conde Lemos, y Andrade, Virrey de Nápoles; $y$ dexando..." ${ }^{43}$.

Mera relación de autores que en muy pocos casos encuentran reflejada su doctrina a lo largo del tratado y que nos refuerzan en la idea de la intención clara de Fray Lorenzo de mostrar sus conocimientos sobre la materia, a los que como Pedro de la Peña ponen en duda su saber ${ }^{44}$.

En relación con el objetante de la Peña se encuentra el capitulo dedicado en la segunda parte a comentar mediante "unas notas que hago en un libro nueuo que ha salido de medidas de bouedas" el libro de Juan de Torija (1624-1666): Breve tratado de todo género de bouedas regulares, y irregulares, execución de obrarlas y medirlas con singularidad y modo moderno, obseruando los preceptos canteriles de los Maestros de Arquitectura, Madrid, 1661. Pedro del Val; pues según Fray

a: Ibıdem. Cap. 1. pág. 3. : “... de las cinco ordenes digo he de hazer estampa de cinco Autores: esto ha de ser escogiendo los cinco mejores a misaber, y entender. Según lo que de la tal orden demuestra, y dize; y la causa desto, y lo que me obliga es, que ay muchas personas curiosas, que con fin de su curiosidad compran estos libros, y es bien que por diseño vean alguna cosa que los aliente y aficione al exercicio, y tambien mancebos, si acaso no tuuieren otro libro sino este, con èl, y con lo poco estampado del podran traçar con mas facilidad de todos los Autores las ordenes que cada uno escriue...".

43 Ibidem. Cap. 49, pág. 179.

44. Los capitulos 2 a 5 de la segunda parte del Arte y Uso de Arquitectura están dedicados a contestar a las treinta y cuatro objeciones que Pedro de la Peña interpuso ante el Consejo Real a la primera parte de la obra 
Lorenzo, Torija, yerno De la Peña, habría plagiado un manuscrito de su suegro, que a su vez lo habria hecho del Libro de traças de cortes de piedras de Alonso de Vandelvira, (1575-1580 aproximadamente) ${ }^{45}$. La verdad es que tanto el enfoque, estereotómico del libro de Vandelvira, y dedicado a la ejecución y medida de bóvedas del de Torija, como los temas tratados, desmienten la "maliciosa» interpretación del agustino ${ }^{46}$.

Solo cabe añadir las numerosísimas referencias que a lo largo de los dos volúmenes del tratado se hace de los clásicos griegos y latinos y que como en tantas obras de la época representan los lugares culturales comunes de las élites.

25 VAndelvira. Alonso de, "Libro de traças de cortes de piedras" (Manuscrito de la Escuela de Arquitectura de Madrid). Ed. facsimil de G. Barbe-Coquelín de Lisle. "Tratado de Arquitectura de Madrid". Albacete, 1977. En WIEBENSON: obra citada, pág. ¿38.

${ }^{46}$ Veáse la reseña del libro de Torija realizada por Fernando Marías en: WIEBENSON, D., obra citada, pags. 246-248. 